
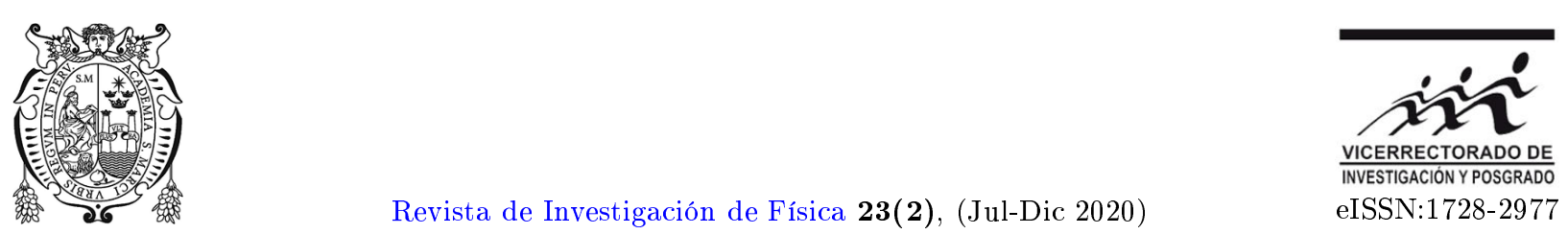

\title{
Un estudio con técnicas físicas de tres piezas minerales pertenecientes al Museo de Historia Natural de la UNMSM
}

\author{
María L. Cerón ${ }^{* 1}$, Jorge A. Bravo ${ }^{1}$ y Felipe A. Reyes ${ }^{1}$ \\ ${ }^{1}$ Laboratorio de Análisis de Suelos y Laboratorio de Arqueometría, Facultad de Ciencias Físicas, \\ Universidad Nacional Mayor de San Marcos, Lima, Perú
}

Recibido 02 noviembre 2020 - Aceptado 21 diciembre 2020

\begin{abstract}
Resumen
Mediante el uso de técnicas analíticas físicas, estudiamos tres piezas minerales pertenecientes al Museo de Historia Natural de la Universidad Nacional Mayor de San Marcos. Nuestro objetivo es obtener detalles sobre su composición elemental y estructural. Específicamente, hemos utilizado técnicas como la fluorescencia de rayos X de dispersión de energía, la difractometría de rayos X, la espectroscopía Mössbauer, la microscopía óptica metalográfica y la microscopía electrónica de barrido. Esta última la usamos en conjunto con la espectroscopía de rayos $\mathrm{X}$ de dispersión de energía. Los resultados obtenidos indican que las muestras MHN-09 y MHN-11 se clasifican como impactitas. Esto demuestra que hubo un impacto meteorítico en Huánuco hace muchos años. También encontramos que la muestra MHN03 es una muestra magnética de acuerdo a los resultados obtenidos por fluorescencia de rayos $\mathrm{X}$ de dispersión de energía y espectroscopía Mössbauer.
\end{abstract}

Palabras clave: Museo de Historia Natural, difracción de rayos x, espectroscopia Mössbauer..

\section{A study on three mineral pieces of the Natural History Museum at the UNMSM by using physical techniques}

\begin{abstract}
By using analytical physical techniques, we studied three mineral pieces belonging to the Natural History Museum at the San Marcos National University. Our aim is to get details concerning their elemental and structural composition. Specifically, we have utilized techniques such as energy dispersive X-ray fluorescence, X-ray diffractometry, and Mössbauer spectroscopy, light optical microscopy and scanning electron microscopy. We used this last one along with energy dispersive X-ray spectroscopy. The obtained results indicate that samples MHN-09 and MHN-11 are impactites. This means that there was a meteorite impact on Huánuco a long time ago. Likewise, we found that sample MHN03 is magnetic in accordance with the results obtained by energy dispersive X-ray fluorescence and Mössbauer spectroscopy.
\end{abstract}

Keywords: Natural History Museum, X-rays diffraction, Mössbauer spectroscopy..

\section{Introducción}

La Universidad Nacional Mayor de San Marcos (UNMSM) cuenta con un Museo de Historia Natural, el cual es una institución académica que fue fundada el 28 de febrero de 1918. Esta entidad colecta, investiga y pone en exhibición especímenes y piezas del patrimonio natural del Perú y del mundo. Específicamente, este museo tiene varias divisiones, siendo la División de Geociencias la que guarda las piezas que hemos estudiado. La exdirectora del museo Dra. Betty Millán Salazar junto con la bióloga Ysabel Prado Velazco nos proporcionaron las muestras que hemos estudiado. Estas muestras fueron recolectadas durante viajes de campo por el fallecido geólogo Luis Guillermo Morales Serrano, exdecano de la otrora Facultad de Geología de la Universidad Nacional Mayor de San Marcos.

En la literatura existen numerosos estudios sobre el patrimonio arqueológico peruano. Específicamente, podemos encontrar estudios sobre piezas de museos perua- 
nos (Schaedel \& Shimada 1982, Saucedo-Segami 2011, Engel 1957). Sin embargo, sólamente existe un estudio sobre las muestras del Museo de Historia Natural de la UNMSM utilizando técnicas físicas. Se trata del trabajo de Cerón \& Bravo (2019), quienes estudiaron dos muestras del Museo de Historia Natural (MHN-08 y MHN-09) usando fluorescencia de rayos $\mathrm{X}$ de dispersión de energía, difractometría de rayos $\mathrm{X}$, espectroscopía Mössbauer de transmisión y microscopía óptica metalográfica. Sin embargo, en ese estudio ellos no reportaron estudios con microscopía electrónica de barrido.

\section{Materiales y métodos}

\section{Preparación de las muestras}

Primeramente, debemos enfatizar que estamos utilizando la numeración registrada previamente por el personal del Museo de Historia Natural de la UNMSM. Por lo tanto, la numeración de las tres piezas no es correlati- va (ver Tabla 1). Así, las muestras fueron pesadas en el mismo laboratorio del Museo de Historia Natural. A continuación, para analizarlas por diferentes técnicas físicas, las llevamos al Laboratorio de Análisis de Suelos de la Facultad de Ciencias Físicas. En la Figura 1, mostramos fotografías de las tres piezas estudiadas. Aquí debemos indicar que de la muestra MHN-09 sólo haremos un estudio complementario inédito, pues esta muestra ya fue parcialmente estudiada por Cerón \& Bravo (2019).

Tabla 1: Las tres muestras del Museo Nacional de Historia de la UNMSM y su correspondiente codificación, masa y procedencia. De la muestra MHN-09 se hará un estudio complementario al reportado por Cerón \& Bravo (2019).

\begin{tabular}{|c|c|c|}
\hline Código & Masa $(\mathrm{g})$ & Procedencia \\
\hline MHN-03 & 26.78 & $\begin{array}{c}\text { Desconocida } \\
\text { MHN-09 }\end{array}$ \\
MHN-11 & 19.0 & $\begin{array}{c}\text { Colectada en Yagapasa, distrito de Llata, } \\
\text { provincia de Humalíes, región Huánuco. } \\
\text { Colectada en distrito de Llata, provincia de } \\
\text { Humalíes, región Huánuco. }\end{array}$ \\
\hline
\end{tabular}
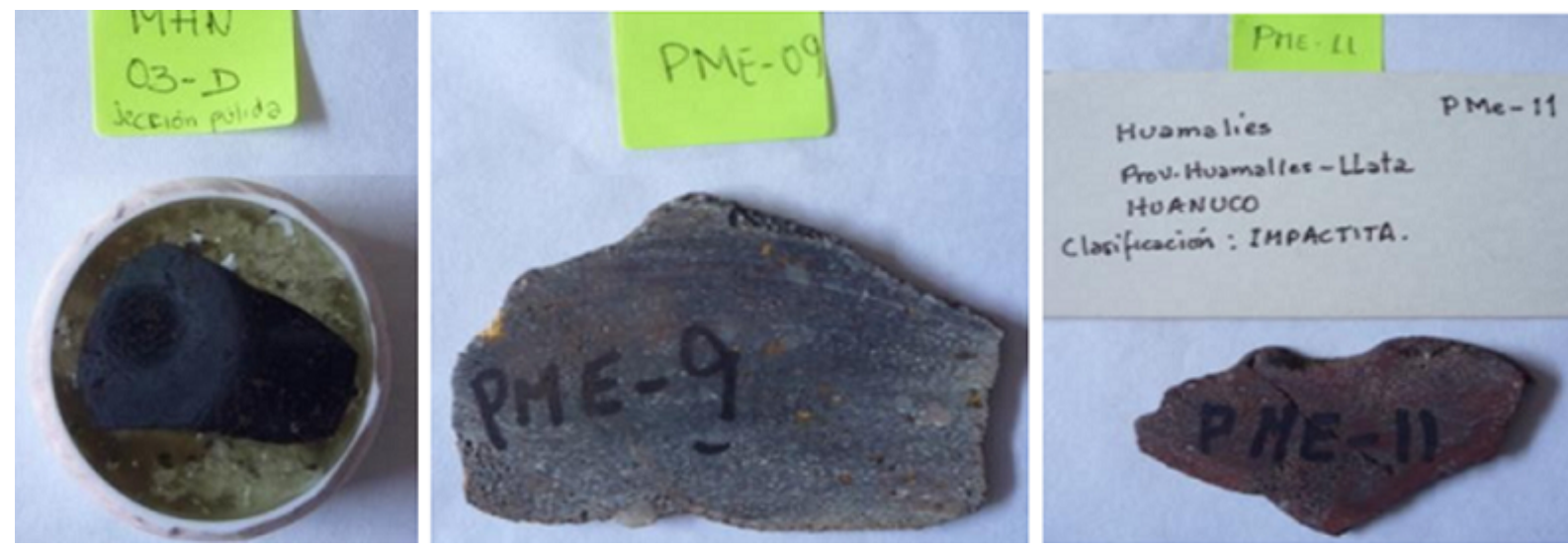

Figura 1: Fotografías de las tres piezas proporcionadas por el Museo de Historia Natural de la UNMSM.

\section{Técnicas físicas utilizadas}

\section{Fluorescencia de rayos $\mathrm{X}$ de dispersión de energía (FRXDE)}

Ésta es una técnica relativamente nueva y no destructiva; se utiliza para identificar elementos químicos con un número atómico mayor de 12 . El análisis de la composición elemental se realizó con un equipo portátil para FRXDE; específicamente, se usó un instrumento de la marca Amptek. Éste utiliza un tubo de rayos X con un ánodo de plata que opera a $30 \mathrm{kV}$ y $15 \mu \mathrm{A}$, y un colimador con un diámetro interno de $2 \mathrm{~mm}$ para colimar el haz de rayos $\mathrm{X}$ incidente.

\section{Difractometría de rayos X (DRX)}

En lo concerniente al equipo, se ha utilizado el difractómetro Bruker, modelo D8-Focus, con radiación $\mathrm{Cu}-\mathrm{K} \alpha$ $(20 \mathrm{kV}, 40 \mathrm{~mA})$ con $\lambda=1.54178 \AA$ y un goniómetro vertical. El ángulo de barrido fue de $4^{\circ}<2 \theta<70^{\circ}$ y el avance fue de $0.02^{\circ} /$ paso con $3 \mathrm{~s} /$ paso.

\section{Espectroscopia Mössbauer (EM)}

Hemos utilizado un espectrómetro convencional Mössbauer con una fuente de ${ }^{57} \mathrm{Co}$ en una matriz de rodio con una actividad promedio de $9 \mathrm{mCi}$ aproximadamente. Para ajustar los espectros, usamos el programa NORMOS creado por R. A. Brand, en las versiones para sitios cristalinos y para distribución de campos (Brand 
2002). Asimismo hemos utilizado 512 y 1024 canales para almacenar los espectros Mössbauer.

\section{Microscopía óptica metalográfica (MOM)}

Hemos utilizado un microscopio metalográfico Euromex Mic. 1060/62, proporcionado por el Laboratorio de Aleaciones Metálicas y Cristales Reales de la Facultad de Ciencias Físicas. Previamente al uso del microscopio, la superficie de las muestras mineralógicas se había desbarbado y luego, pulido. Asimismo, atacamos la superficie con el reactivo Nital al $2 \%$ con diferentes tiempos de exposición. Nital es una solución de alcohol y ácido nítrico, que generalmente se utiliza para atacar químicamente los metales.

\section{Microscopía electrónica de barrido junto con un análisis de dispersión de energía de rayos $\mathrm{X}$ (MEB-DiRX)}

Estos análisis lo hemos realizado en la Facultad de Ciencias Biológicas de la UNMSM. Así, hemos utilizado un microscopio electrónico de barrido acoplado con un analizador de dispersión de energía de rayos X. El microscopio electrónico de barrido que utilizamos es un modelo FEI INSPECT S50 con un accesorio para detectar la composición elemental mediante la dispersión de energía de rayos $\mathrm{X}$.

\section{Resultados y Discusión}

\section{Análisis por Fluorescencia de rayos $\mathrm{X}$ de dis- persión de energía}

\section{Análisis cualitativo y semicuantitativo}

Por un lado, el análisis cualitativo consiste en identificar los elementos presentes a partir de los rayos $\mathrm{X}$ característicos que se manifiestan en los espectros como picos de distintas amplitudes (Donais \& George 2018).

Por otro lado, los resultados de los análisis cuantitativos se obtuvieron ajustando el espectro FRXDE experimental con un programa que simula espectros FRXDE basado en el modelo de parámetros fundamentales. El mencionado programa está escrito en FORTRAN y simula la geometría experimental y los procesos físicos que determinan el espectro de fluorescencia de rayos $\mathrm{X}$. La función de distribución de los rayos $\mathrm{X}$, que incluye componentes tanto continuos como discretos, se ha calibrado mediante la dispersión de estos rayos $\mathrm{X}$ primarios mediante muestras conocidas. La eficacia del programa se ha verificado utilizando muestras de referencia. La incertidumbre estimada en estas medidas de concentraciones elementales es aproximadamente del $10 \%$, dependiendo del número atómico del elemento y aspectos estadísticos.
Nuestras muestras fueron analizadas irradiando una región uniforme, con el objetivo de que las mediciones no sean destructivas.

Los análisis cualitativos y cuantitativos se llevaron a cabo en las muestras MHN-03 y MHN-11 (los datos de MHN-09 ya están publicados en Cerón \& Bravo (2019)). La Tabla 2 muestra los correspondientes resultados; la muestra MHN-03 es claramente magnética debido a la alta concentración de hierro $(60.31 \%)$. A continuación, reportamos datos sobre las dos muestras: (a) Muestra MHN-03. Tiene una sección pulida. Se registró con una tasa de conteo de $1349 \mathrm{cts} / \mathrm{s}$ a 3.7 A y $29.2 \mathrm{keV}$. (b) Muestra MHN-11. Se registró con una tasa de conteo de $1243 \mathrm{cts} / \mathrm{s}$ a $10.11 \mu \mathrm{A}$ y $29.5 \mathrm{keV}$.

Tabla 2: Análisis elemental cuantitativo por FRXDE para dos muestras del Museo de Historia Natural de la UNMSM (para MHN-09, los datos ya están publicados en Cerón \& Bravo (2019). Se muestran las concentraciones por porcentaje de masa de cada elemento.

\begin{tabular}{|c|c|c|c|c|c|}
\hline \multirow{2}{*}{ Elemento } & \multicolumn{2}{|c|}{ Muestra } & \multirow{2}{*}{ Elemento } & \multicolumn{2}{|c|}{ Muestra } \\
\hline & MHN-03 & MHN-11 & & MHN-03 & MHN-11 \\
\hline $\mathrm{Si}$ & 0.00 & 28.65 & $\mathrm{Ni}$ & 0.17 & 0.00 \\
\hline $\mathrm{P}$ & 0.00 & 0.00 & $\mathrm{Cu}$ & 0.36 & 0.07 \\
\hline $\mathrm{Cl}$ & 0.00 & 0.00 & $\mathrm{Zn}$ & 0.03 & 0.03 \\
\hline $\mathrm{K}$ & 0.04 & 0.17 & As & - & - \\
\hline $\mathrm{Ca}$ & 0.21 & 0.05 & $\mathrm{Rb}$ & 0.45 & - \\
\hline $\mathrm{Ti}$ & 0.14 & 0.18 & $\mathrm{Sr}$ & - & 0.02 \\
\hline $\mathrm{V}$ & 0.22 & 0.05 & $\mathrm{Zr}$ & - & 0.02 \\
\hline $\mathrm{Cr}$ & 0.25 & 0.10 & $\mathrm{La}$ & 0.08 & 0.15 \\
\hline Mn & 0.93 & 0.29 & $\mathrm{Ba}$ & - & - \\
\hline $\mathrm{Fe}$ & 60.31 & 8.27 & W & - & - \\
\hline \multicolumn{2}{|c|}{ Subtotal } & \multicolumn{2}{|c|}{ MHN-03 (63.19) } & \multicolumn{2}{|c|}{ MHN-11 (38.05) } \\
\hline \multicolumn{2}{|c|}{ Otros $(\mathrm{Z}<14)$} & \multicolumn{2}{|c|}{ MHN-03 (36.81) } & \multicolumn{2}{|c|}{ MHN-11 (61.95) } \\
\hline \multicolumn{2}{|c|}{ TOTAL \% } & \multicolumn{2}{|c|}{ MHN-03 (100) } & \multicolumn{2}{|c|}{ MHN-11 (100) } \\
\hline
\end{tabular}

\section{Análisis por diffracción de rayos $\mathrm{X}$}

Para la identificación estructural de los minerales y las fases cristalográficas presentes en las muestras estudiadas, se ha utilizado la técnica de DRX (Cullity 1978). Además, en relación con los nombres de los minerales encontrados en rocas, debemos señalar que utilizaremos las abreviaturas estandarizadas por Whitney \& Evans (2010). También, debemos indicar que a causa de recibir encapsulada la muestra MHN-03, no la pulverizamos. En consecuencia no hicimos un análisis de DRX para esta muestra. Asimismo, para MHN-09, el difractograma de rayos X ya fue publicado en Cerón \& Bravo (2019). A continuación, tenemos los resultados de DRX para la muestra MHN-11.

\section{Muestra MHN-11}

En la Figura 2, observamos los picos principales de las fases mineralógicas goethita (Gth), hematita (Hem), caolinita (Kln), cuarzo (Qz) y stishovita (Sti). Hay picos 
anchos y superpuestos de Hem + Gth; los picos característicos principales corresponden a hematita, mientras que goethita aparece con picos de menor intensidad. En $2 \theta=24.11^{\circ}$ y $2 \theta=54.08^{\circ}$ sólo aparece hematita con picos anchos y en $2 \theta=55.44^{\circ}$, goethita aparece sólo con picos bien definidos. Por lo tanto, inferimos que la fase bien cristalizada correspondería a goethita. Hematita se- ría una fase pobremente cristalizada.

También en la Figura 2, observamos picos de quarzo en $2 \theta=20.89^{\circ}, 26.66^{\circ}$ y $39.46^{\circ}$. En $2 \theta=45.8^{\circ}$ encontramos Qz + Sti, donde el aporte de stishovita es mayor, mientras que el del cuarzo es mínimo. Se observa caolinita con sus picos principales característicos.

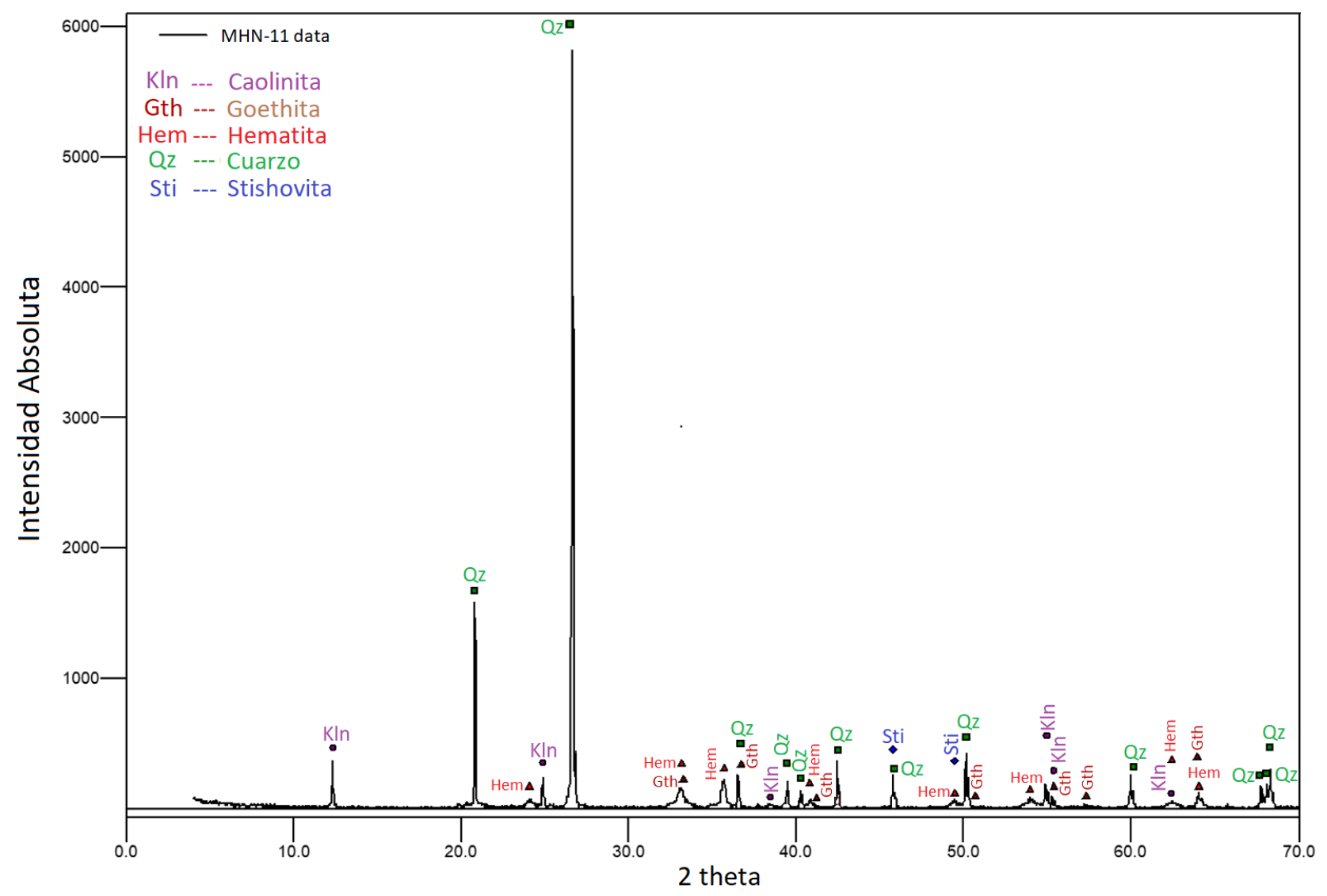

Figura 2: Difractograma de rayos X de la muestra MHN-11.

A continuación, en relación con las fases mineralógicas que hemos identificado, la Tabla 3 enumera los archivos que se encuentran en la base de datos del ICCD (International Center for Diffraction Data), cuya página web es ww. icdd.com.

Tabla 3: Archivos ICCD de las fases mineralógicas identificadas en la muestra estudiada por DRX.

\begin{tabular}{|c|c|c|}
\hline Muestra & Mineral & Código \\
\hline & Cuarzo & $78-2315$ \\
MHN-11 & Caolinita & $78-1996$ \\
& Goethita & $29-713$ \\
& Hematita & $87-1164$ \\
& Stishovita & $82-1646$ \\
\hline
\end{tabular}

Análisis por espectroscopia Mössbauer a temperatura ambiente y a temperatura del helio líquido

En la Tabla 4, a temperatura ambiente, tenemos los parámetros hiperfinos de las muestras mineralógicas MHN-03 y MHN-11.

Tabla 4: A temperatura ambiente, parámetros hiperfinos de dos muestras mineralógicas del $\mathrm{Mu}-$ seo de Historia Natural. Para MHN-9, los datos ya fueron publicados en Cerón \& Bravo (2019). 


\begin{tabular}{|c|c|c|c|}
\hline \multirow{2}{*}{ Sitio } & \multirow{2}{*}{ Parámetro } & \multicolumn{2}{|c|}{ Muestra } \\
\cline { 3 - 4 } & & MHN-03 & MHN-11 \\
\hline \multirow{4}{*}{ Hematita S1 (I) } & $\delta(\mathrm{mm} / \mathrm{s})$ & $0.30(1)$ & $0.296(1)$ \\
& $2 \varepsilon / \Delta \mathrm{E}_{\mathrm{Q}}(\mathrm{mm} / \mathrm{s})$ & $-0.19(1)$ & $-0.23(1)$ \\
& $\mathrm{B}_{\mathrm{hf}}(\mathrm{T})$ & $52.22(2)$ & $51.2(3)$ \\
& $\mathrm{A}(\%)$ & 100 & 43.63 \\
& $\Gamma(\mathrm{mm} / \mathrm{s})$ & 0.32 & 0.27 \\
\hline \multirow{3}{*}{ Hematita S2 (II) } & $\delta(\mathrm{mm} / \mathrm{s})$ & - & $0.31(5)$ \\
& $2 \varepsilon / \Delta \mathrm{E}_{\mathrm{Q}}(\mathrm{mm} / \mathrm{s})$ & - & $-0.22(9)$ \\
& $\mathrm{B}_{\mathrm{hf}}(\mathrm{T})$ & - & $48.2(4)$ \\
& $\mathrm{A}(\%)$ & - & 25.57 \\
& $\Gamma(\mathrm{mm} / \mathrm{s})$ & - & 0.56 \\
\hline \multirow{4}{*}{ Hematita S3 (III) } & $\delta(\mathrm{mm} / \mathrm{s})$ & - & $0.30(5)$ \\
& $2 \varepsilon / \Delta \mathrm{E}_{\mathrm{Q}}(\mathrm{mm} / \mathrm{s})$ & - & $-0.217(9)$ \\
& $\mathrm{B}_{\mathrm{hf}}(\mathrm{T})$ & - & $50.12(4)$ \\
& $\mathrm{A}(\%)$ & - & 30.80 \\
& $\Gamma(\mathrm{mm} / \mathrm{s})$ & - & 0.38 \\
\hline
\end{tabular}

\section{Muestra MHN-03}

Como ya se ha indicado, esta muestra no fue pulverizada. Así, hemos utilizado la espectroscopia Mössbauer (Dickson \& Berry 1986), pero a través del método de reflexión. Esta modalidad es no destructiva y nos permite observar una capa superficial de unos $10 \mu \mathrm{m}$ de espesor; sin embargo ella no permite una alta resolución estadística. Los resultados se muestran en la Figura 3, donde se observa la presencia de un sólo sexteto magnético, con $\mathrm{B}_{\mathrm{hf}}=52.2 \mathrm{~T}$.

\section{Muestra MHN-11}

En la Figura 4, tenemos el espectro a temperatura ambiente. Observamos fases minerales asignadas a tres sextetos magnéticos S1, S2 y S3. De los tres, S1 y $\mathrm{S} 2$, correspondientes a óxidos de $\mathrm{Fe}^{3+}$, son asignados a hematita, con campos $\mathrm{B}_{\mathrm{hf}}=51.2 \mathrm{~T}$ y $\mathrm{B}_{\mathrm{hf}}=48.2 \mathrm{~T}$, respectivamente. Observamos que los picos de S1 son delgados; por lo tanto, se encuentran en una fase más cristalizada mientras que S2 presenta picos anchos. Esto nos permitiría definir a S2 como hematita de baja cristalinidad. El tercer sexteto, S3, con campo $\mathrm{B}_{\mathrm{hf}}=$ $50.1 \mathrm{~T}$, también correspondería a hematita que constituía parte de bauxita, la cual es una roca sedimentaria formada por la meteorización y puede contener más de un óxido de hierro. Esta roca sedimentaria sufrió un impacto meteorítico convirtiéndose en impactita. Hacemos hincapié que MHN-11 no es una muestra total de bauxita. MHN-11 es una impactita (Koeberl et al. 2012 ).

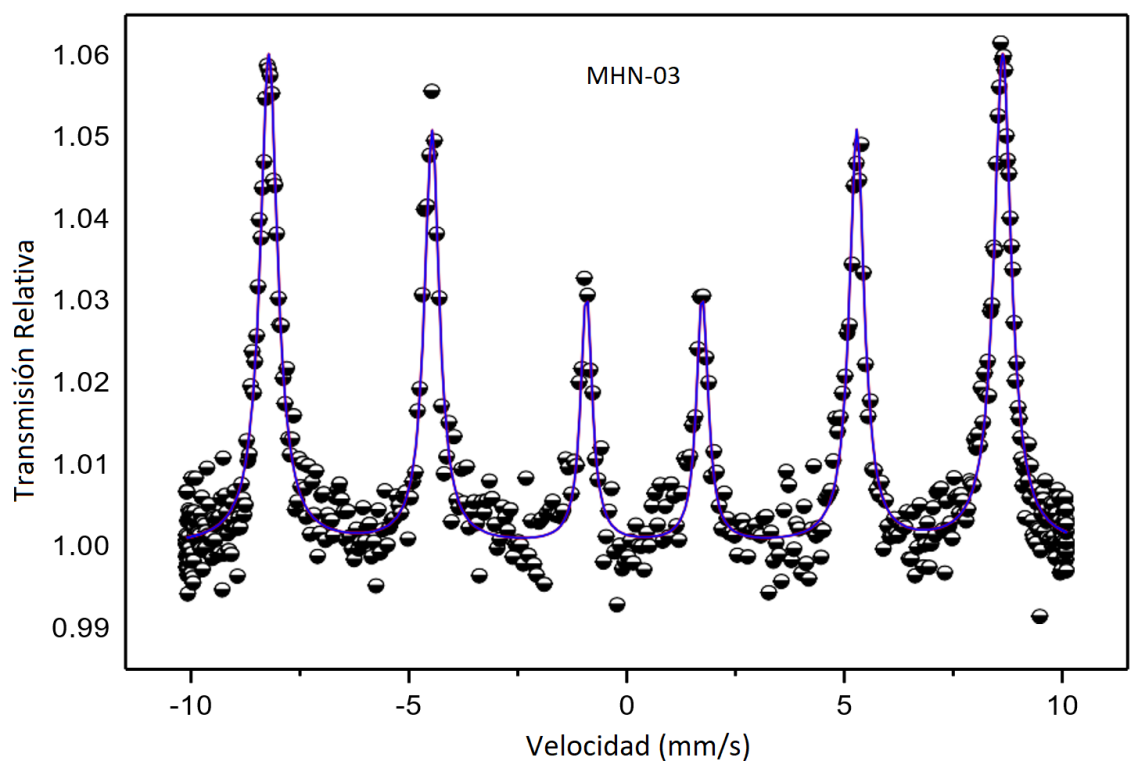

Figura 3: Para la muestra MHN-03, espectro Mössbauer por dispersión registrado a temperatura ambiente.

En los espectros por dispersión a la temperatura de helio líquido, como se observa en la Figura 5, observamos que S1 y S2 aumentan sus campos a medida que disminuye la temperatura. Interpretamos que estos minerales han sufrido un ordenamiento magnético, y S3, con $\mathrm{B}_{\mathrm{hf}}=$ $55 \mathrm{~T}$, corresponde al mineral hematita. Los parámetros hiperfinos correspondientes se muestran en la Tabla 5. 


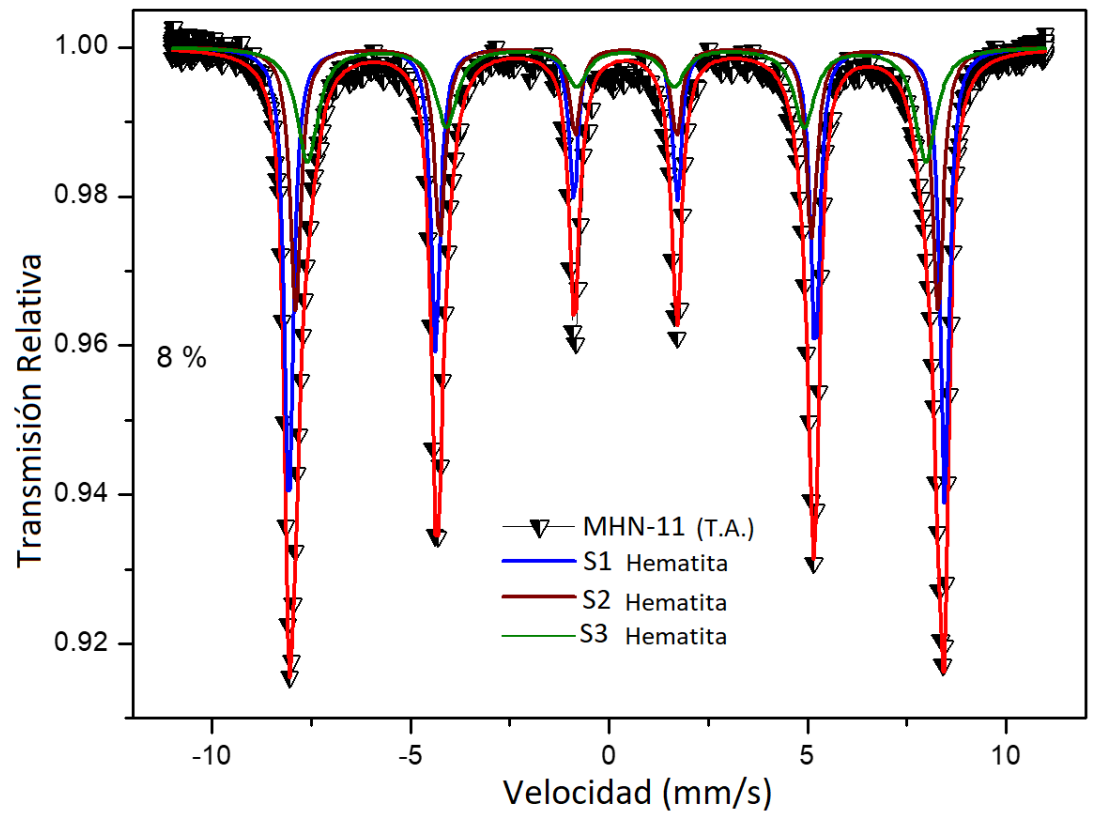

Figura 4: Espectros Mössbauer de la muestra MHN-11 registrados a temperatura ambiente.

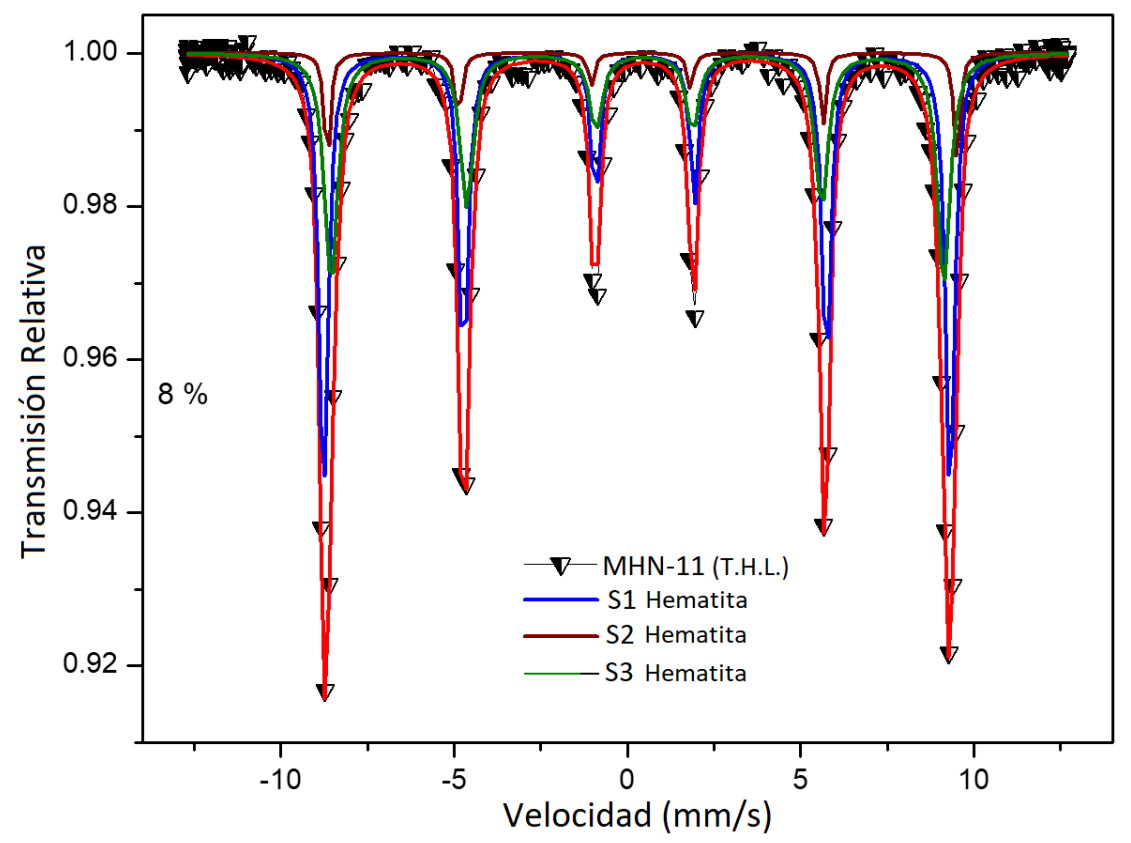

Figura 5: Espectros Mössbauer de la muestra MHN-11 registrados a temperatura de helio liquido. 
Tabla 5: A temperatura de helio líquido, parámetros hiperfinos de la muestra MHN-11 del Museo de Historia Natural.

\begin{tabular}{|c|c|c|}
\hline \multicolumn{1}{|c|}{ Sitio } & \multirow{2}{*}{ Párametro } & Muestra \\
\cline { 3 - 3 } & & MHN-11 \\
\hline \multirow{4}{*}{ Hematita S1 (I) } & $\delta(\mathrm{mm} / \mathrm{s})$ & 0.386 \\
& $2 \varepsilon / \Delta \mathrm{E}_{\mathrm{Q}}(\mathrm{mm} / \mathrm{s})$ & -0.218 \\
& $\mathrm{~B}_{\mathrm{hf}}(\mathrm{T})$ & 55.99 \\
& $\mathrm{~A}(\%)$ & 52.99 \\
& $\Gamma(\mathrm{mm} / \mathrm{s})$ & 0.31 \\
\hline & $\delta(\mathrm{mm} / \mathrm{s})$ & 0.403 \\
Hematita S2 (II) & $2 \varepsilon / \Delta \mathrm{E}_{\mathrm{Q}}(\mathrm{mm} / \mathrm{s})$ & -0.039 \\
& $\mathrm{~B}_{\mathrm{hf}}(\mathrm{T})$ & 56.13 \\
& $\mathrm{~A}(\%)$ & 8.50 \\
& $\Gamma(\mathrm{mm} / \mathrm{s})$ & 0.45 \\
\hline \multirow{3}{*}{ Hematita S3 (III) } & $\delta(\mathrm{mm} / \mathrm{s})$ & 0.384 \\
& $2 \varepsilon / \Delta \mathrm{E}_{\mathrm{Q}}(\mathrm{mm} / \mathrm{s})$ & -0.213 \\
& $\mathrm{~B}_{\mathrm{hf}}(\mathrm{T})$ & 54.95 \\
& $\mathrm{~A}(\%)$ & 38.58 \\
& $\Gamma(\mathrm{mm} / \mathrm{s})$ & 0.39 \\
\hline
\end{tabular}

\section{Análisis por microscopia electrónica de barrido junto a un análisis por dispersion de rayos $\mathrm{x}$ (MEB-DiRX). Análisis por mi- croscopía óptica metalográfica (MOM)}

\section{Muestra MHN-09}

Los resultados se pueden apreciar en la Figura 6, donde se observa claramente las formas eudrales de la muestra, las cuales fueron registradas con MOM a una escala de 400X. Se observa la presencia de minerales como cuarzo; también se observa la forma de estrías y pliegues de esta muestra. Esto sería consecuencia de los aumentos de presión y temperatura sobre la superficie terrestre, en la cual se encontraba originalmente la muestra, provocados probablemente por un fuerte impacto meteorítico.

En la Figura 7, tenemos los resultados por MEB y espectroscopía DiRX, para un punto dado. Observamos las concentraciones de los elementos presentes en la muestra, en especial silicio que forma parte de la composición del cuarzo y los polimorfos observados por difracción de rayos X. Estos últimos fueron transformados por las altas presiones y temperaturas, generadas sólo durante una posible colisión de un impacto meteoritico (Tormo Cifuentes 2013).

\section{Muestra MHN-11}

En la Figura 8a observamos la fotografía de la muestra con MOM, con una escala de $100 \mathrm{X}$ (izquierda) y se especifican los extremos, donde observamos fracturas, cambiando abruptamente la textura y el tamaño de grano. Las Figuras 8b y 8c muestran los resultados con MEB, asignando la presencia de abundante cuarzo y hematita a $800 \mathrm{X}$ y $1500 \mathrm{X}$, respectivamente. En la Figura 9 se registró un punto, donde los resultados por espectroscopía MEB y DiRX corroboran el porcentaje de concentración de los elementos silicio y oxigeno. Sin embargo, no se observa la presencia del elemento hierro, lo cual no significa que no esté presente sino que por esta técnica se tomó un punto donde hay abundancia de silicio y no de hierro.

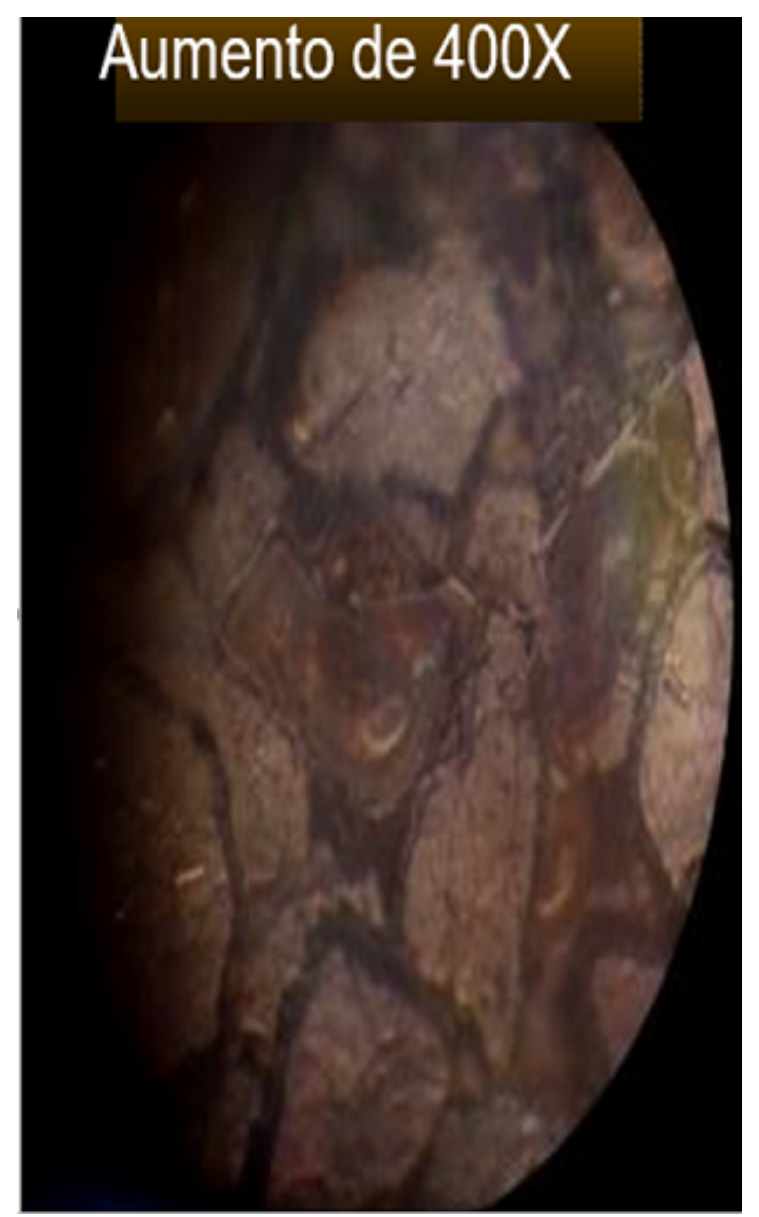

Figura 6: Muestra MHN-09 observada por microscopia óptica metalográfica con aumento de 400X. Se observan formas eudrales y la presencia de cuarzo. 

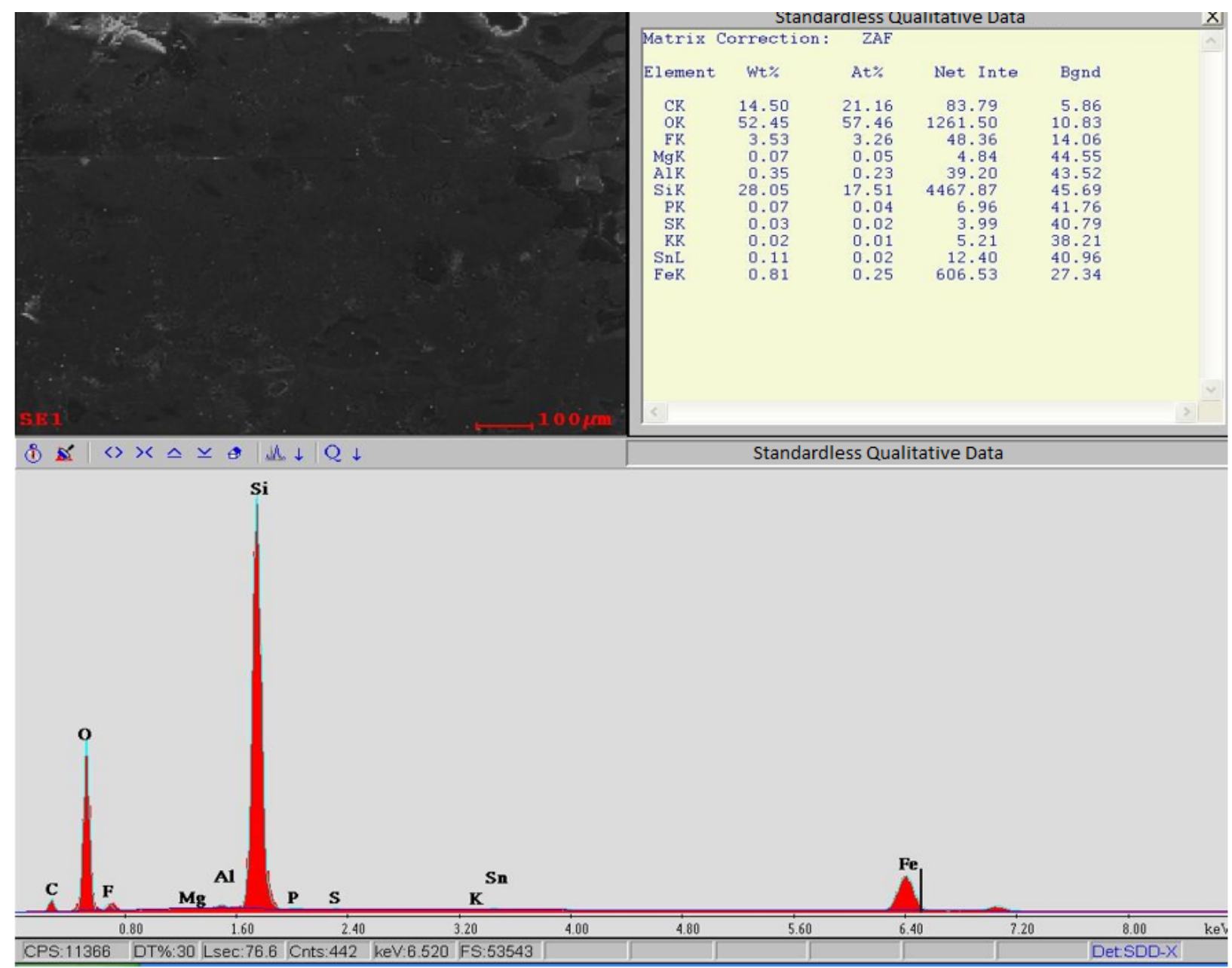

Figura 7: Para MHN-09, resultados por microscopia electrónica de barrido (parte superior izquierda) y espectroscopia de rayos X por dispersión de energía (parte inferior). También, se muestran las concentraciones de los elementos (parte superior derecha).

\section{Conclusiones}

Los resultados obtenidos con las técnicas EM, DRX y FRXDE, complementados con MOM y MEB, permitieron concluir que la muestra MHN-11 se clasifica como impactita. Esto se debe a la presencia de coesita y stishovita, así como a ringwoodita, que es un polimorfo de olivino. Por lo tanto, esto demuestra que hubo un impacto meteorítico que ocurrió en Huánuco hace muchos años.

Asimismo, para la muestra MHN-09, el estudio complementario inédito con MOM y MEB permite corroborar que se trata de una impactita. Finalmente, la muestra MHN-03 es una muestra magnética conforme a los resultados obtenidos por FRXDE y EM.

\section{Agradecimientos}

Primeramente, agradecemos al Ing. Guillermo Morales Serrano (in memoriam) por haber facilitado datos acerca del origen de procedencia de las tres muestras estudiadas. Asimismo, agradecemos a la Dra. Betty Millán Salazar, exdirectora del Museo de Historia Natural de la UNMSM, y a la Biol. Ysabel Prado Velazco por proporcionarnos las muestras utilizadas en este trabajo.

También agradecemos, por todo el apoyo brindado, al personal del Laboratorio de Análisis de Suelos, Laboratorio de Arqueometría, Laboratorio de Cristalografía de Rayos X y Laboratorio de Aleaciones Metálicas y Cristales Reales. 

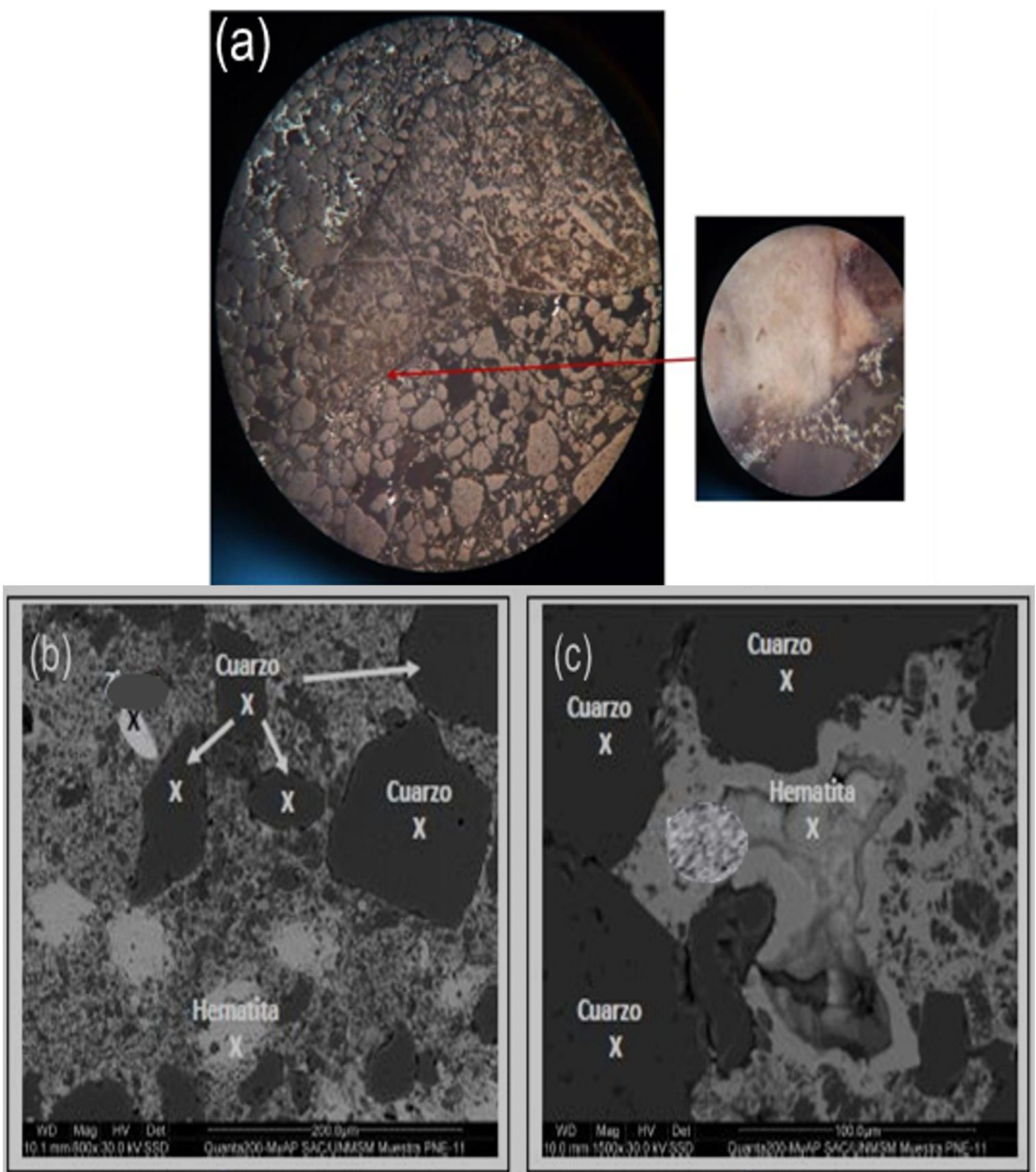

Figura 8: Muestra MHN-11. (a) Resultados con MOM, la textura y el tamaño de los granos se observan a 100X. (b) y (c) Resultados por MEB donde se observan abundantes fragmentos minerales como el cuarzo y la hematita, registrados con un aumento de $800 \mathrm{X}$ y $1500 \mathrm{X}$, respectivamente. Observamos la textura del gap entre el cuarzo cementado con hematita. 


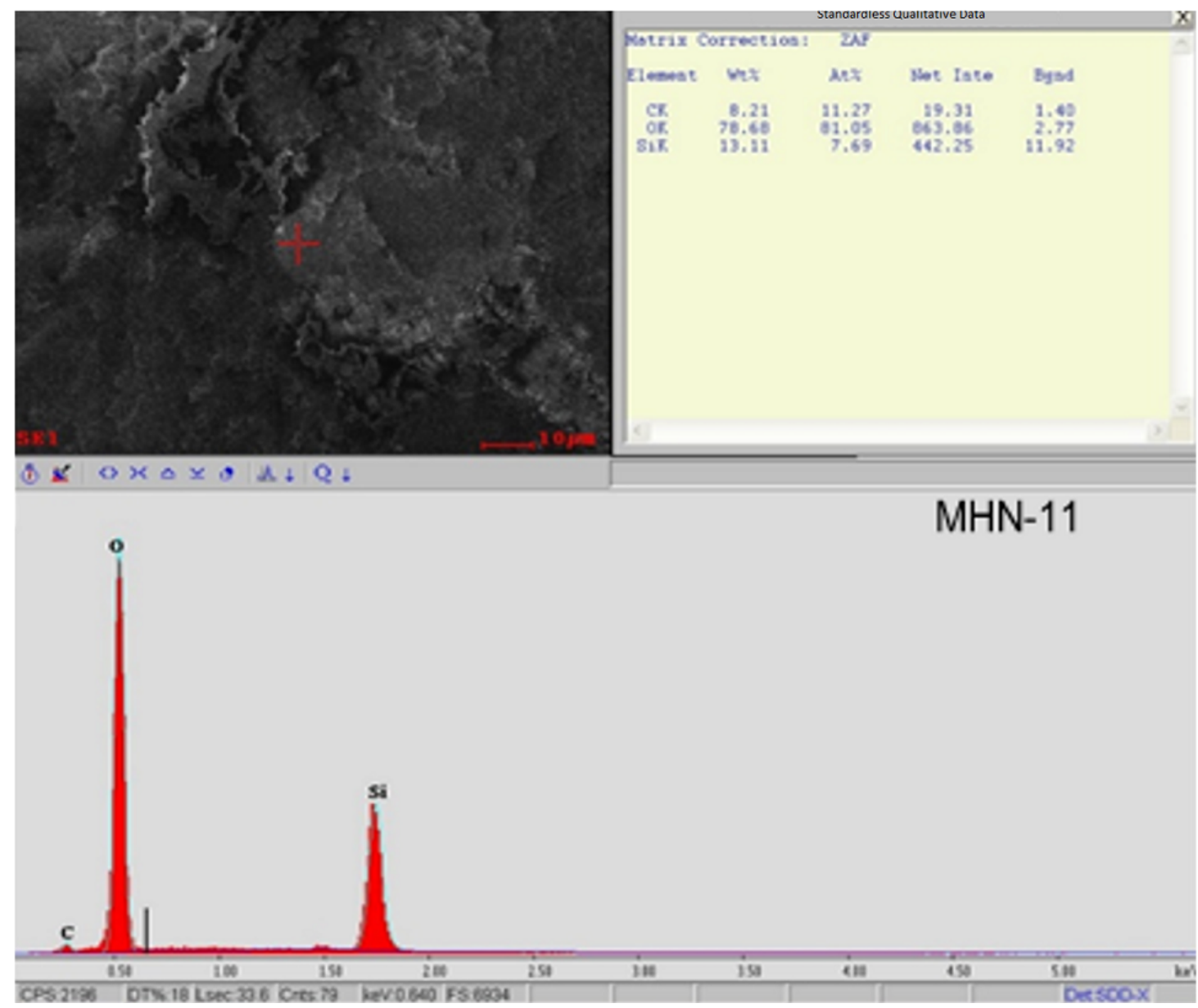

Figura 9: Para MHN-11, resultados por microscopia electrónica de barrido (parte superior izquierda) y espectroscopia de rayos X por dispersión de energía (parte inferior). Observamos nítidamente la presencia de los picos de silicio y oxígeno, elementos componentes del cuarzo. Asimismo, se muestran las concentraciones de los elementos (parte superior derecha).

\section{Referencias}

[Bra02] Brand, R. (2002). NORMOS Mössbauer Fitting Program, Universität Dortmund, Dortmund, Germany. https://physics-astronomy-manuals.wwu. edu/Wissel $\% 20$ NORMOS $\% 2090 \% 20$ Manual.pdf

[Cer19] Cerón, M. y Bravo, J. (2019). Characterization of magnetic iron phases in impactites by Mössbauer spectroscopy, Hyperfine Interact 240, 90. https:// doi.org/10.1007/s10751-019-1616-5

[Cul78] Cullity, B. (1978). Elements of X-Ray Diffraction. Second Edition, Addison-Wesley.
[Dic86] Dickson, D. and Berry F. (1986). Mössbauer Spectroscopy, Cambridge University Press.

[Don18] Donais, M. and George, D. (2018). X-ray fluorescence spectrometry and its applications to archaeology : an illustrated guide. Series: Materials characterization and analysis collection, Momentum Press.

[Eng57] Engel, F. (1957). Early sites on the Peruvian coast. Southwestern Journal of Anthropology, 13(1), pp 54-68. doi : 10.1086/soutjanth.13.1.3629157

[Koe12] Koeberl, C., Claeys, P., Hecht, L. and McDonald, I. (2012). Geochemistry of Impactites. Ele- 
ments 8(1): pp 37-42, 2012. http://www.we.vub. ac . be $/ \sim \operatorname{dglg} /$ Web/Claeys/pdf/Koeberl-2012.pdf

[Sau11] Saucedo-Segami, D. (2011). Looking for an Identity: Archaeologists, Local Communities, and Public Archaeology in Peru. In: K. Okamura and A. Matsuda (eds) New Perspectives in Global Public Archaeology. Springer, New York, NY. https://doi.org/ 10.1007/978-1-4614-0341-8_19

[Sch82] Schaedel, R. and Shimada, I. (1982). Peruvian
Archaeology, 1946-80: An analytic overview. World Archaeology 13:3, pp 359-371.

[Tor13] Tormo, L. (2013). Estudio de meteoritos singulares por técnicas espectroscópicas y de luminiscencia no destructivas. Tesis doctoral. Universidad Autónoma de Madrid. https://dialnet.unirioja.es/ servlet/tesis? codigo $=41897$

[Whi10] Whitney, D. and Evans, B. (2010). Abbreviations for names of rock-forming minerals, American Mineralogist, Vol. 95, pp 185-187. 